\title{
Upregulation of Foxo3a protects neurons against hypoxia-ischaemia injury
}

Rongjia Lu ${ }^{1}$, Huajie Cai ${ }^{2}$, Yu Liao ${ }^{3}$, Keman Liao ${ }^{4}$, Jianhua Wang ${ }^{1}$, Bin Liao ${ }^{1}$, Shaofu Zhou ${ }^{1}$, Shaoping Chen ${ }^{1}$, Renhua Huang ${ }^{5}$, Ping Zheng ${ }^{6}$, Sunhai Zhou ${ }^{4}$

${ }^{1}$ Department of Neurosurgery, Longyan People Hospital, Longyan, Fujian Province, China

2Department of Geriatrics, Renji Hospital, School of Medicine, Shanghai Jiao Tong University, Shanghai, China

${ }^{3}$ Department of Endocrinology and Metabolism, South Campus, Ren Ji Hospital,

School of Medicine, Shanghai Jiao Tong University, Shanghai, China

${ }^{4}$ Department of Neurosurgery, Renji Hospital, School of Medicine, Shanghai Jiao Tong University, Shanghai, China

${ }^{5}$ Department of Radiation Oncology, Renji Hospital, School of Medicine, Shanghai Jiao Tong University, Shanghai, China

${ }^{6}$ Department of Neurosurgery, Shanghai Pudong New area People's Hospital,

Shanghai, China

Submitted: 1 February 2020

Accepted: 22 May 2020

Arch Med Sci

DOI: https://doi.org/10.5114/aoms.2020.96620

Copyright @ 2020 Termedia \& Banach

\section{Abstract}

Introduction: A series of transcription factors have been profiled in ischaemic stroke. Forkhead box protein $\mathrm{O} 3 \mathrm{a}$ (FoxO3a), which belongs to the family of transcription factors, is characterised by a forkhead DNA-binding domain. However, the role of FoxO3a and circFoxO3, which is encoded from FOXO3 gene in stroke, remains unelucidated.

Material and methods: A rat model of middle cerebral artery occlusion (MCAO)/ reperfusion was applied, and rat neuroblastoma B35 cells were treated with cobalt chloride to mimic cellular hypoxia in vitro. FoxO3a (or circFoxO3a) was manipulated to assess the infarction volume and apoptotic proteins.

Results: Brain infarction was enlarged in ischaemia/reperfusion compared with simple ischaemia treatment. The protein level of FoxO3a increased significantly in MCAO and subsequently decreased following reperfusion. Increased FoxO3a expression was also found in $\mathrm{CoCl}_{2}$ treatment at $24 \mathrm{~h}$ and 48 h. Downregulation of FoxO3a significantly promoted apoptosis, while upregulation of FoxO3a reduced apoptosis. Further biochemical analysis demonstrated that protein expression level of caspase-3, caspase-9, and $\mathrm{Bax}$ were upregulated following FoxO3a inhibition but downregulated following FoxO3a overexpression. Moreover, upregulated FoxO3a level was consistent with enhanced circFoxO3 expression both in vivo and in vitro. Conclusions: Both FoxO3a and circFoxO3 are upregulated in ischaemic stroke, which associates with the apoptosis pathway. Hence, FoxO3a and circFoxO3 might be protective factors against hypoxia/ischaemia-induced neuronal damage.

Key words: ischaemia/reperfusion, FoxO3a, circFoxO3, hypoxia, apoptosis.

\section{Introduction}

Cerebral ischaemic stroke has long been recognised as a prevalent and serious neurological disease associated with high mortality and morbidi-

\author{
Corresponding authors: \\ Sunhai Zhou MD \\ Department \\ of Neurosurgery \\ Renji Hospital \\ School of Medicine \\ Shanghai Jiao Tong \\ University \\ Shanghai, China \\ E-mail: zhousunhai@alumni. \\ sjtu.edu.cn \\ Ping Zheng MD, PhD \\ Department \\ of Neurosurgery \\ Shanghai Pudong \\ New area People's Hospital \\ Shanghai, China \\ E-mail: ping.zheng@ \\ ukmuenster.de
}


ty [1]. The pathophysiology of ischaemic stroke is complicated and multifactorial [2-4]. There is an urgent need to determine the mechanisms behind stroke pathophysiology. Accumulating evidence has suggested that neuronal apoptosis plays an important role in cell loss following acute ischaemic damage through the activation of various signalling pathways [5-7]. However, the precise mechanism that regulates the activation of apoptotic pathway in the injured brain is yet to be fully elucidated.

FoxO3a, member of the forkhead box O (FoxO) transcription factor family, is a downstream transcriptional factor of the PI3K/AKT pathway [8-12]. Once it is phosphorylated by AKT, FoxO3a rapidly translocates from the nucleus to the cytoplasm, where phosphorylated FoxO3a is ubiquitylated and degraded [13-17]. Moreover, FoxO3a can be phosphorylated by IKK and ERK as well as in response to growth factor and insulin stimulation $[15,18]$. Taken together, negative regulation of FoxO3a by phosphokinases is a key mechanism of promoting FoxO3a functions, but downstream molecular events remain elusive.

FoxO3a has multiple functions, most of which are related to ischaemia/hypoxia stress and tumour regulation. FoxO3a was first discovered due to its role in tumour differentiation and proliferation $[16,19-22]$. However, the role of FoxO3a in ischaemia and hypoxia is controversial. Experimental cardiac research indicates that FoxO3a causes cardiomyocyte death $[23,24]$. Another study showed that FoxO3a has protective effects in cardiomyocyte ischaemia-reperfusion (I-R) [25], and similar reports have been made in the kidney [26], liver [27], and lung [28]. At present, there have been few studies on the role of FoxO3a in cerebral ischaemia/hypoxia, especially in I-R. The total amount of FoxO3a is unchanged when cerebral ischaemia and hypoxia occur, but the level of dephosphorylated FoxO3a (d-FoxO3a) increases, leading to the entry of FoxO3a into the nucleus, which further results in an increase in apoptosis-related proteins and thereby promotes apoptosis [29-31]. It has also been suggested that I-R changes FoxO3a levels in the hippocampal CA1 region [32]. Protein expression of phosphorylated FoxO3a (p-FoxO3a) decreased during cerebral ischaemia and hypoxia but increased $4 \mathrm{~h}$ after reperfusion [33]. Considering the complex biological behaviour of FoxO3a in ischaemia/hypoxia-related cerebral injury, we aim to investigate its underlying mechanisms.

CircFoxO3 is a newly identified circRNA generated from gene $\mathrm{FOXO} 3$, which locates on chromosome $6 q 21$. CircFoxO3 was reported to be downregulated in breast cancer [34] and non-small cell lung cancer [35] but upregulated in glioblastoma [36]. Functionally, circFoxO3 acts as a powerful tumour regulator through sponging certain miRNAs targeting the parental transcript, FoxO3 [37]. Considering the multiple functions of protein FoxO3a in tumour regulation, circFoxO3 may act as suppressor or activator depending on certain tumour types. William et al. reported that circFoxO3 was upregulated in heart samples from aged patients [38] and that it promoted cardiac senescence. However, the role of circFoxO3 in ischaemic stroke, which has not been reported, is of interest.

In the present study, we investigated whether the expression levels of FoxO3a and circFoxO3 changed during I/R. We further explored whether FoxO3a protected hypoxic neuronal cells against apoptosis, as well as its synergistic effect with circFoxO3.

\section{Material and methods}

\section{Animals and groups}

Male Sprague-Dawley (SD) rats (purchased from the Experimental Animal Centre of Renji Hospital, Shanghai Jiaotong University School of Medicine) weighing 250-300 g were housed in a climate-controlled room, with five rats per cage, and with food and water provided ad libitum on a 12 : $12 \mathrm{~h}$ light:dark cycle. The animal study protocol was approved by the Ethics Committee for Animal Experiment and conducted according to the Guidelines for Animal Experimentation of Renji Hospital, Shanghai Jiaotong University School of Medicine (20170223-001). Non-retrospective ethical approval for the animal experiments was obtained for the study. A total of 18 rats were randomly assigned to the following groups: sham group (operated on but not subjected to occlusion), MCAO group (operated on and subjected to occlusion for $90 \mathrm{~min}$ ), and MCAO/reperfusion group (operated on and subjected to occlusion for 90 mins before being reperfused for $30 \mathrm{~min}$ ).

\section{MCAO/reperfusion model construction}

Briefly, SD rats were intraperitoneally anaesthetised with $50 \mathrm{mg} / \mathrm{kg}$ pentobarbital sodium (VWR International, Ltd., Poole, England) and then attached to the operating table. The external carotid artery (ECA) and its branches were isolated and ligated, and a 3-0 monofilament nylon suture (Beijing Shadong Industrial Corp., China) with a heat-treated, rounded tip was introduced into the right internal carotid artery (ICA) via the ECA until slight resistance was achieved. The incisions were then sutured, and the rats were left alone for $90 \mathrm{~min}$. After that, the rats were anaesthetised again as described above before the suture was withdrawn to facilitate reperfusion. The rats were finally euthanised with excess pentobarbital sodium, and whole brain tissues were removed. The extracted brain tissues were program prepared into paraffin sections before assessment. The area 
of the infarct volume was analysed using Adobe Photoshop CS4 Extended Version 11.0.1 (Adobe Systems Inc., San Jose, CA, USA). The infarct volume of each slice was calculated using the following equation: infarct volume $=$ infarct area of the slice $\times$ thickness of the slice $(2 \mathrm{~mm})$. The total infarct volume was calculated by adding all the infarct volumes of each slice.

\section{Cell culture and treatment with $\mathrm{CoCl}_{2}$ to mimic hypoxia}

The rat neuroblastoma B35 cell line (ATCC, Manassas, VA) has been established as a suitable model to study hypoxia-injury (HI)-induced neuronal injury $[35,39]$. The cells were maintained at $37^{\circ} \mathrm{C}$ in Dulbecco's modified Eagle's medium (DMEM) (Gibco, Carlsbad, CA, USA) containing $10 \%$ heat-inactivated foetal bovine serum (Gibco) in a humidified atmosphere with $5 \% \mathrm{CO}_{2}$. $\mathrm{COCl}_{2}$ was diluted in DMEM and sterilised through a 0.2 micron filter prior to use. According to previous studies, $\mathrm{CoCl}_{2}$ was further diluted in DMEM to a final concentration of $250 \mu \mathrm{mol}$ for use in subsequent experiments $[35,39]$. The cultures were divided into control $(\mathrm{O} \mathrm{h})$ or $\mathrm{CoCl}_{2}$-treated (treated for 24,48 , or 72 h) groups.

\section{Reverse transcription-quantitative PCR}

Total RNA was extracted from the cells or ischaemic penumbra brain tissue in the MCAO/reperfusion group and MCAO group or the equivalent brain tissue in the sham group using TRIzol (Invitrogen; Thermo Fisher Scientific, Inc.) according to the manufacturer's instructions. An equal amount of total RNA was used for first-strand cDNA synthesis using an oligo-dT primer and M-myeloblastosis virus reverse transcriptase $\mathrm{XL}$ (Promega). The synthesised first-strand CDNA $(2 \mu \mathrm{l})$ was used for $P C R$. The RT-PCR primers used to amplify FoxO3a were as follows: F: 5'- AAGGGAAGGAGCCGAGGTA-3'; R: 5'-CGACTCTGTGGCTCGAACTCT-3'. SYBR Green PCR Master Mix (ABI) was used for real-time RT-PCR. $\beta$-Actin was used as an internal reference control. The RT-PCR primers used to amplify rat $\beta$-actin were as follows: F: $5^{\prime}$ - CGTAAAGACCTCTATGCCAACA-3'; R: 5'- AGCCACCAATCCACACAGAG-3'.

\section{Western blot analysis}

Total proteins were extracted from the cells in cell lysis buffer (50 mM Tris- $\mathrm{HCl}$ ( $\mathrm{pH} 8.0$ ), $120 \mathrm{mM}$ $\mathrm{NaCl}, 0.5 \% \mathrm{NP}-40,1 \mathrm{mM} \mathrm{PMSF}$ ), and the protein concentration was determined by the BCA method. The proteins $(30 \mu \mathrm{g})$ were subjected to $8-10 \%$ SDS-polyacrylamide gel electrophoresis and transferred onto Hybond ECL membranes (Amersham). The membranes were incubated for $1 \mathrm{~h}$ at room temperature in blocking buffer $(5 \%$ skimmed milk in TBS-T) and then incubated with the appropriate antibodies (rabbit anti-FoxO3a (cat. no. 12829, Cell Signalling Technology; 1 : 1000) and mouse anti- $\beta$-actin (Millipore; 1 : 10,000)) overnight at $4^{\circ} \mathrm{C}$. After being washed with TBS-T, the membranes were incubated with horseradish peroxidase-conjugated anti-rabbit or anti-mouse antibody ( $1: 10,000$; Sigma) for $2 \mathrm{~h}$ at room temperature. Western blot detection reagents (Odyssey) were used to detect proteins on the membranes. The corresponding bands were semi-quantitatively analysed by ImageJ (NIH, version 1.8.0).

\section{Lentiviral vector-mediated gene knockdown or overexpression}

FoxO3a knockdown and overexpression vectors were constructed by Hanyin Co. (Shanghai, China). Specifically, human FoxO3a or FoxO3a target sequences were separately cloned into the pMSCV-IRES-GFP plasmids for construction of FoxO3a overexpression or knockdown recombinant lentivirus vectors. The knockdown sequence of FoxO3a was: 5'-GCTCTTGGTGGATCATCAA-3'. To construct circFoxO3 overexpression (OE) plasmids, a basic sequence (flanked by $\mathrm{Hxol}$ and Agel) was synthesised by qRT-PCR. Primer sequence: F: 5'-ATTGTCCATGGAGACAGCCCGCCG-3'; R: 5'-GTGGGGAACTTCACTGGTGCTAAG-3'. A small spacer sequence containing two restriction enzyme sites, HindIII and Sall, was added for the insertion of the circRNA fragment. The recombinant and negative control (NC) lentiviruses (Hanyin Co. Shanghai, China) were prepared and tittered to $10^{9} \mathrm{TU}$ (transfection unit)/ml. After $48 \mathrm{~h}$, the knockdown or overexpression efficiency was confirmed via quantitative real-time polymerase chain reaction (qRT-PCR). To obtain stable cell lines, the cells were seeded in sixwell plates at a density of $2 \times 10^{5}$ cells per well. The cells were then infected with the same viral titre with $8 \mu \mathrm{g} / \mathrm{ml}$ Polybrene on the following day. Approximately $72 \mathrm{~h}$ after viral infection, the culture medium was replaced with selection medium containing $4 \mu \mathrm{g} / \mathrm{ml}$ puromycin for selecting cell lines with stable expression of targeted sequence. The cells were then cultured for at least 14 days. Puromycin-resistant cells were amplified in medium containing $2 \mu \mathrm{g} / \mathrm{ml}$ puromycin for 7 to 9 days and then transferred to a medium without puromycin.

\section{Cell apoptosis analysis}

Apoptosis was determined by detecting the translocation of phosphatidylserine to the cell surface using an annexin and DAPI apoptosis detection kit (BD Inc., USA). Rat neuroblastoma B35 cells were treated with $\mathrm{CoCl}_{2}(250 \mu \mathrm{mol})$ at the indicated time. Then, the cells were harvested, washed twice in cold PBS, and resuspended in annexin V-fluo- 
A

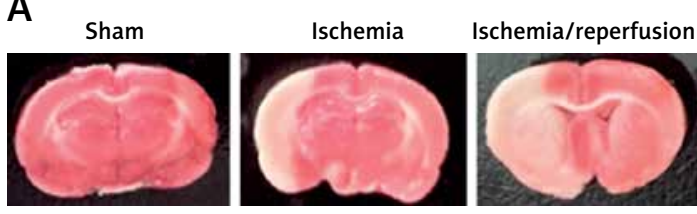

C

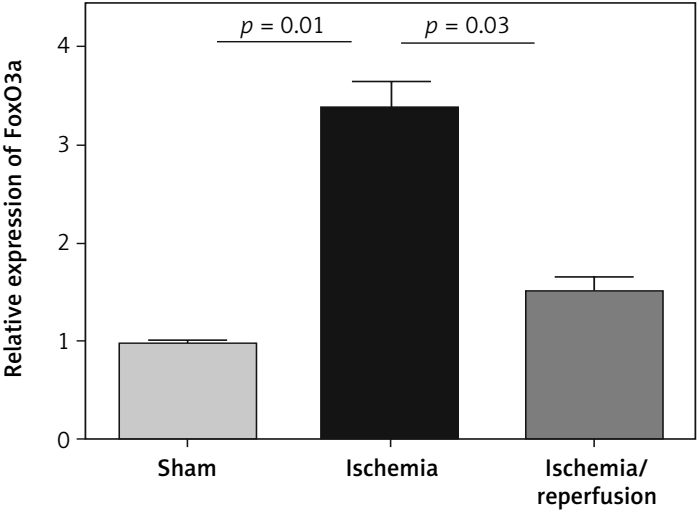

$\mathrm{E}$

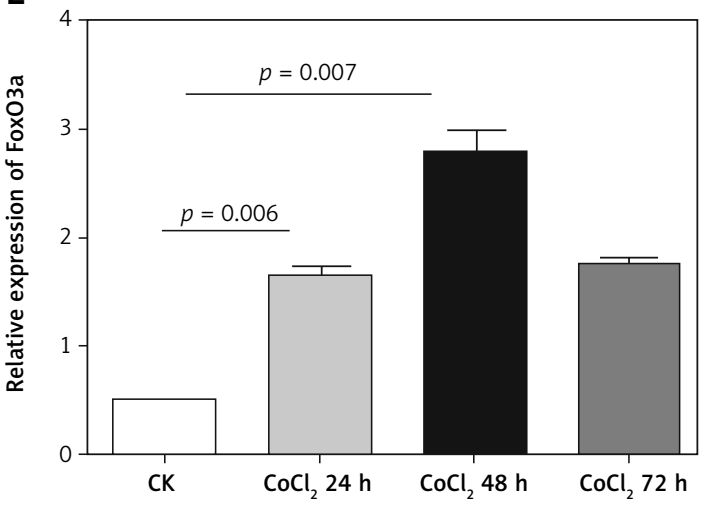

B

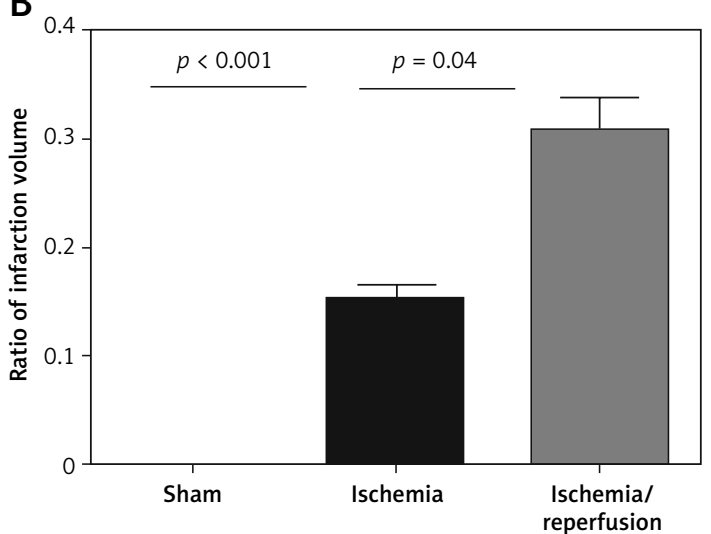

D

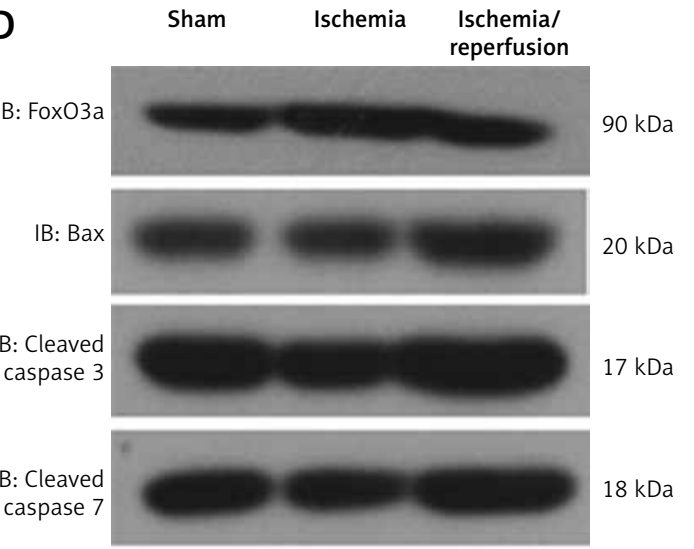

IB: Actin

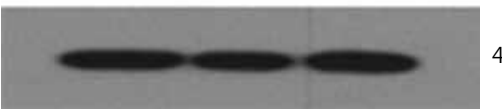
$43 \mathrm{kDa}$

$\mathrm{F}$

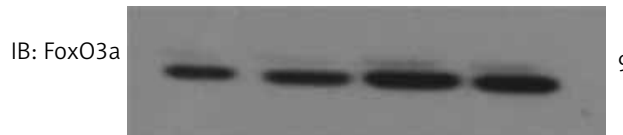

$90 \mathrm{kDa}$

IB: Bax

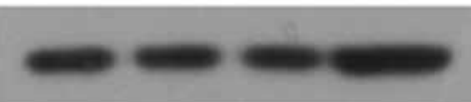

$20 \mathrm{kDa}$

IB: Cleaved

caspase 3

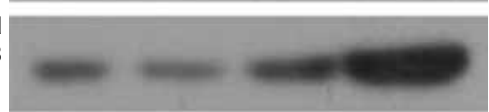

$17 \mathrm{kDa}$

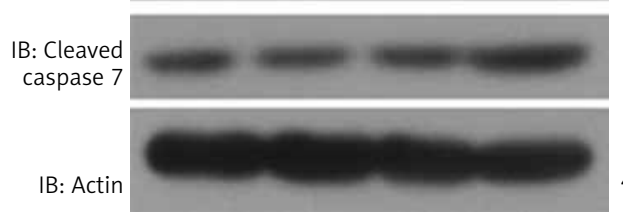

$18 \mathrm{kDa}$

$43 \mathrm{kDa}$

Figure 1. Increased expression of FoxO3a after $\mathrm{H} / \mathrm{I}$ exposure in vivo and in vitro. A - Gross examination of the infarct brain tissues in rats in the sham, MCAO, and MCAO/reperfusion groups. B - Measurements of infarct volume in three groups by TTC staining, expressed as the ratio of the infarct volume to the total volume. $C$ - Relative expression levels of FoxO3a in the sham, MCAO, and MCAO/reperfusion groups. $\mathbf{D}$ - Expression levels of FoxO3a, Bax, caspase-3, and caspase-7 in the sham, MCAO, and MCAO/reperfusion groups. E - Relative FoxO3a mRNA expression levels in $\mathrm{B} 35$ cells treated with $\mathrm{CoCl}_{2}$ for $24 \mathrm{~h}, 48 \mathrm{~h}$, and $72 \mathrm{~h}$. F - Expression levels of FoxO3a, Bax, caspase-3, and caspase-7 in B35 cells treated with $\mathrm{CoCl}_{2}$ for $24 \mathrm{~h}, 48 \mathrm{~h}$, and $72 \mathrm{~h}$ 
rescein isothiocyanate (FITC) and $\mathrm{PI}$ for $30 \mathrm{~min}$ in the dark. Cell apoptosis was analysed by using Cell Quest software on a FACSAria flow cytometer (BD Inc., USA). Fluorescence was detected with an excitation wavelength of $480 \mathrm{~nm}$.

\section{Statistical analysis}

Data are presented as the mean \pm the standard error of the mean. A Tukey test was conducted for multiple comparisons in conjunction with oneway analysis of variance. All statistical analyses were performed using SPSS for Windows v. 17.0 (SPSS, Chicago, IL). All results were considered significant with a two-sided $p$-value $<0.05$.

\section{Results}

\section{The increased expression of FoxO3a after $\mathrm{H} / \mathrm{I}$ in vivo and in vitro}

We successfully constructed a rat model of middle cerebral artery occlusion (MCAO)/reperfusion. TTC staining showed that the infarction volume was significantly increased after MCAO and even higher after reperfusion (Figures 1 A, B). Both RT$\mathrm{PCR}$ and western blot revealed that $\mathrm{FoxO} 3 \mathrm{a}$ was significantly upregulated during MCAO and decreased after reperfusion in vivo (Figures 1 C, D).

A

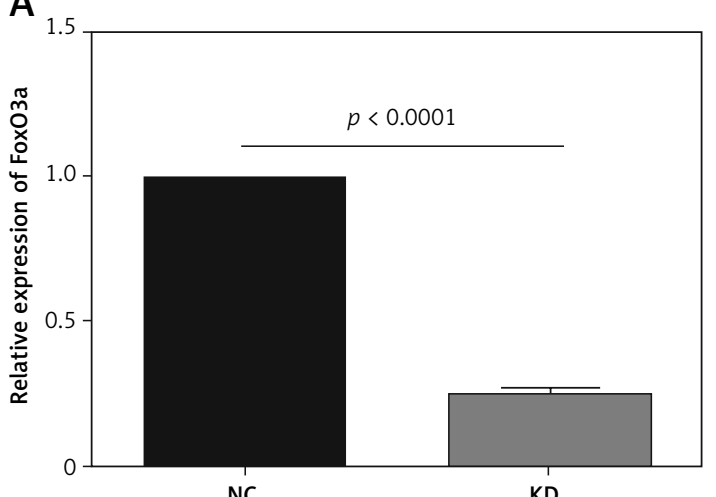

NC

KD

C

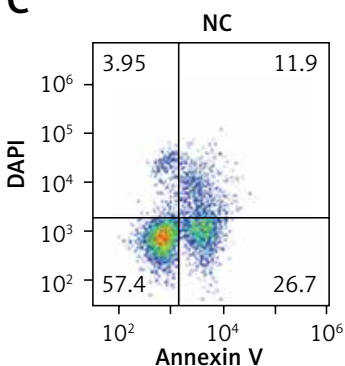

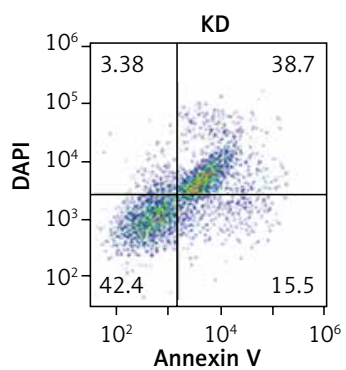

The caspase-3, caspase-7 and Bax levels were also significantly increased in I/R model (Figure $1 \mathrm{D}$ ). Similarly, in $\mathrm{B} 35$ cells treated with $\mathrm{CoCl}_{2}$, the expression of FoxO3a was significantly increased after $24 \mathrm{~h}$, with a peak at $48 \mathrm{~h}$, and decreased at $72 \mathrm{~h}$ (Figures $1 \mathrm{E}, \mathrm{F}$ ), indicating that the expression of FoxO3a was increased after $\mathrm{H} / \mathrm{I}$ both in vivo and in vitro. The caspase- 3 , caspase- 7 , and Bax levels were significantly increased at $72 \mathrm{~h}$ (Figure $1 \mathrm{~F}$ ).

\section{The knockdown of FoxO3a increased} apoptotic neuronal cells in response to hypoxia/ischaemia

To investigate the function of FoxO3a in hypoxia/ischaemia, we constructed stable FoxO3a knockdown B35 cells using shRNA lentivirus. As shown in Figures $2 \mathrm{~A}$ and $\mathrm{B}$, FoxO3a was efficiently inhibited in knockdown (KD) cells compared to negative control (NC) cells (Figures $2 \mathrm{~A}, \mathrm{~B}$ ). An apoptosis assay of these cells after $\mathrm{CoCl}_{2}$ treatment showed that FoxO3a suppression increased the percentage of apoptotic cells compared to that in control cells (Figures 2 C, D). Altogether, these results indicate that the knockdown of FoxO3a in neuronal cells elevated the percentage of apoptotic cells after ischaemia and they imply a neuroprotective effect of FoxO3a.

B
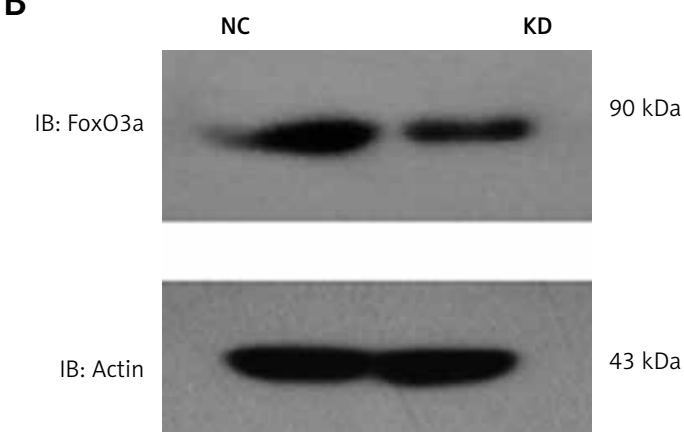

D

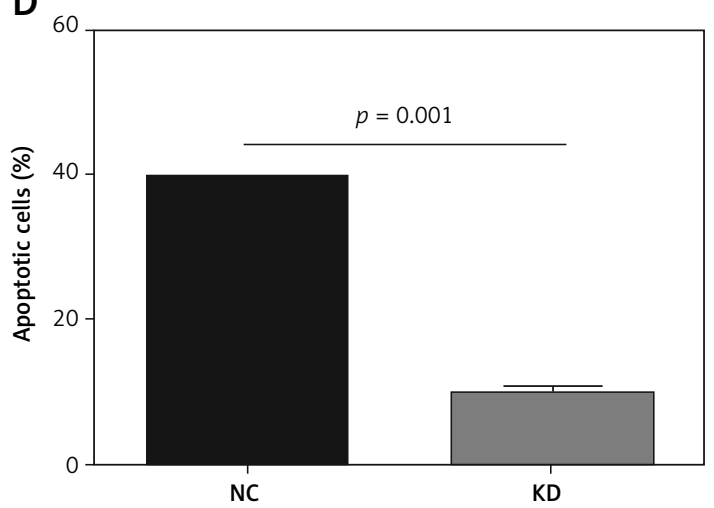

Figure 2. The knockdown of FoxO3a expression increased apoptotic neuronal cells in response to hypoxia/ischaemia. A, B - Relative FoxO3a mRNA and protein expression levels in B35 cells with or without FoxO3a knockdown. C, D - Percentage of apoptotic B35 cells with or without FoxO3a knockdown treated with $\mathrm{CoCl}_{2}$ for $72 \mathrm{~h}$. All the experiments were repeated three times 
The overexpression of FoxO3a reduced apoptotic neuronal cells in response to hypoxia/ischaemia

To further clarify the neuroprotective function of FoxO3a in hypoxia/ischaemia, we constructed stable FoxO3a-overexpressing B35 cells using lentivirus. As shown in Figures $3 \mathrm{~A}$ and $\mathrm{B}$, FoxO3a was efficiently overexpressed in $\mathrm{OE}$ cells compared to negative control (NC) cells (Figures $3 \mathrm{~A}, \mathrm{~B}$ ). An apoptosis assay in these cells after $\mathrm{CoCl}_{2}$ treatment showed that FoxO3a overexpression reduced the percentage of apoptotic cells (Figures 3 C, D). Altogether, these results indicate that the overexpression of FoxO3a in neuronal cells reduced the percentage of apoptotic cells after ischaemia.

The upregulation of FoxO3a suppresses apoptotic protein levels in neuronal cells in response to hypoxia/ischaemia

We further analysed the apoptotic proteins in FoxO3a knockdown or FoxO3a-overexpressing cells. Western blotting verified that the expression of the apoptotic proteins including caspase-3, caspase-9, and Bax were increased after FoxO3a knockdown (Figures 4 A, B). In contrast, the expression of the apoptotic marker caspase-3,

A

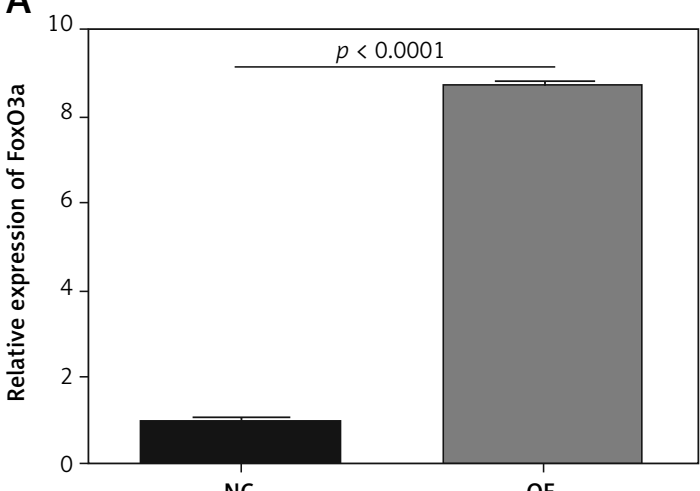

NC

C
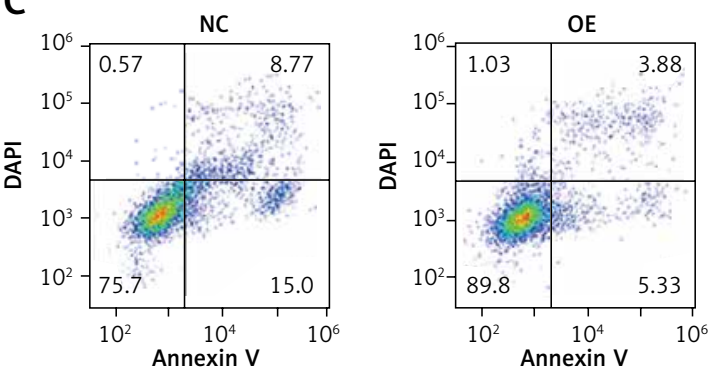

caspase-9, and Bax were significantly reduced after FoxO3a overexpression (Figures 4 C, D). Altogether, these results indicate that FoxO3a protects neuronal cells from hypoxia/ischaemia-induced apoptosis partially through inhibition of the apoptotic pathway.

\section{The upregulation of circFoxO3 enhanced} FoxO3a expression levels in neuronal cells

We further analysed the expression of circFoxO3, and the results showed that consistent with FoxO3a, circFoxO3 was significantly upregulated during MCAO and decreased after reperfusion in vivo (Figure $5 \mathrm{~A}$ ). We thus constructed stable circFoxO3-overexpressing B35 cells using lentivirus (Figure 5 B). Overexpression of circFoxO3 significantly enhanced expression of FoxO3a (Figures $5 \mathrm{C}, \mathrm{D}$ ) indicating that the upregulation of circFoxO3 enhanced FoxO3a expression levels in neuronal cells.

\section{Discussion}

In a rat model of MCAO/reperfusion and in Co$\mathrm{Cl}_{2}$-treated (hypoxia mimetic) rat neuroblastoma cells, this study showed that the circRNA and protein expression of FoxO3a increased after ischae-

B

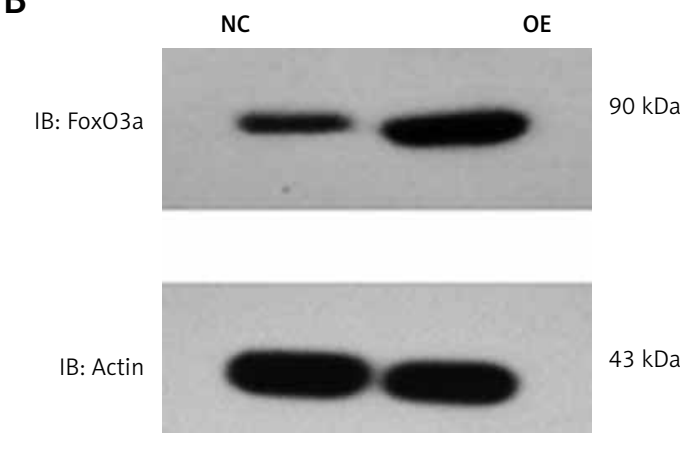

D

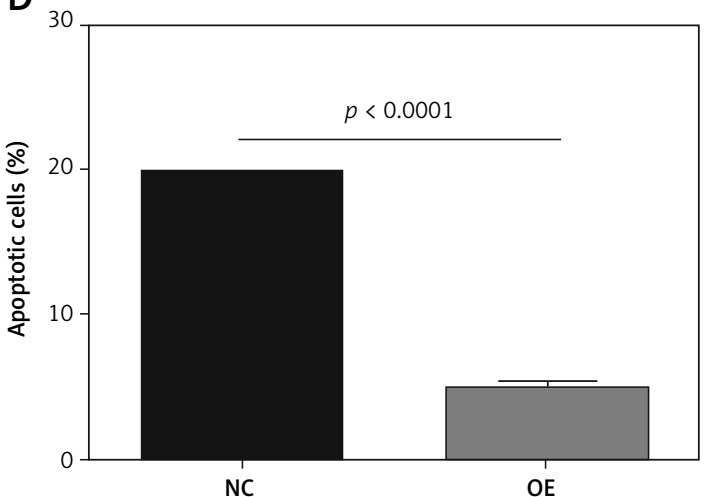

Figure 3. The overexpression of FoxO3a reduced the proportion of apoptotic neuronal cells in response to hypoxia/ ischaemia. A, B - Relative FoxO3a mRNA and protein expression levels in B35 cells with or without FoxO3a overexpression. C, D - Percentage of apoptotic B35 cells with or without FoxO3a overexpression treated with $\mathrm{CoCl}_{2}$ for $72 \mathrm{~h}$. All the experiments were repeated three times 
A
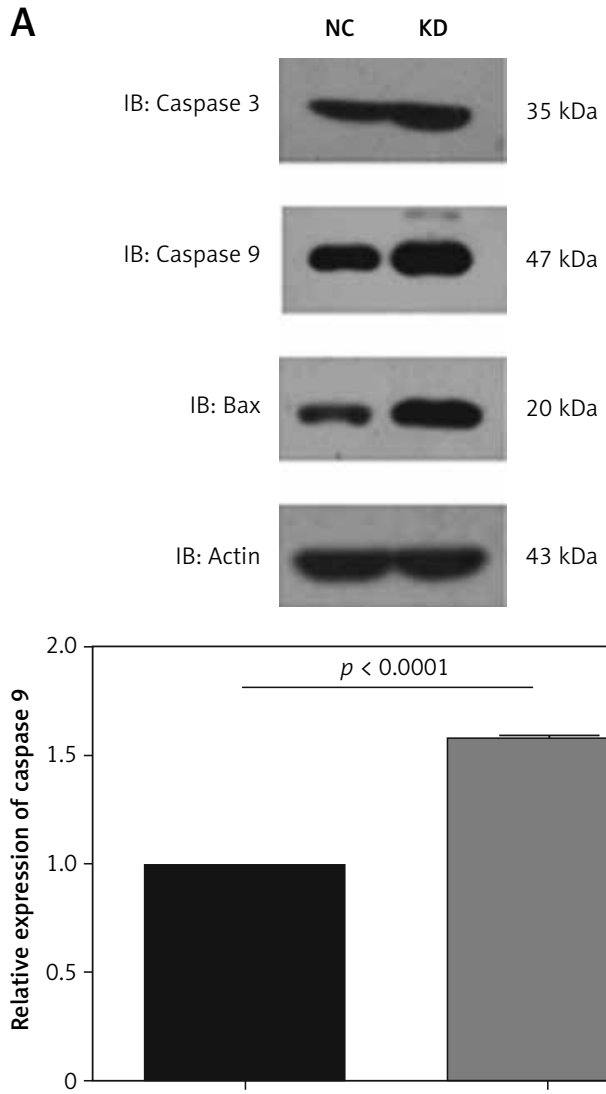

NC

C

IB: Caspase 3

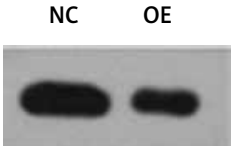

KD

IB: Caspase 9

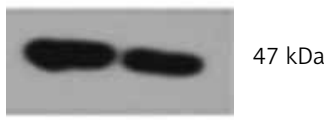

IB: Bax

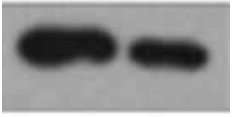

$20 \mathrm{kDa}$

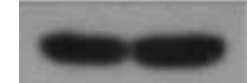

$43 \mathrm{kDa}$

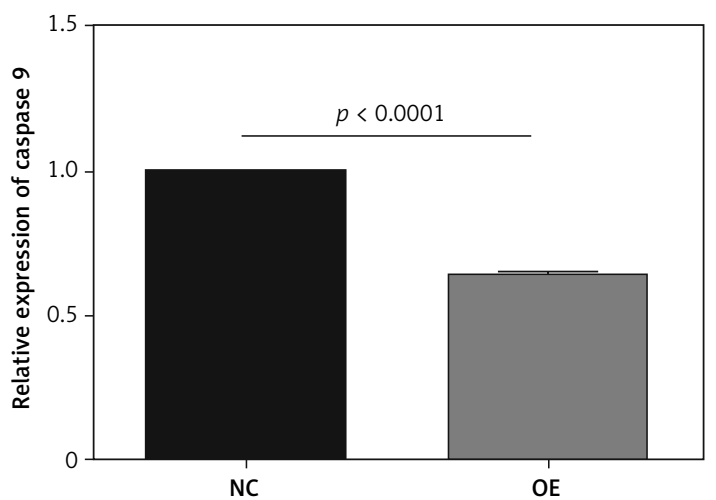

B
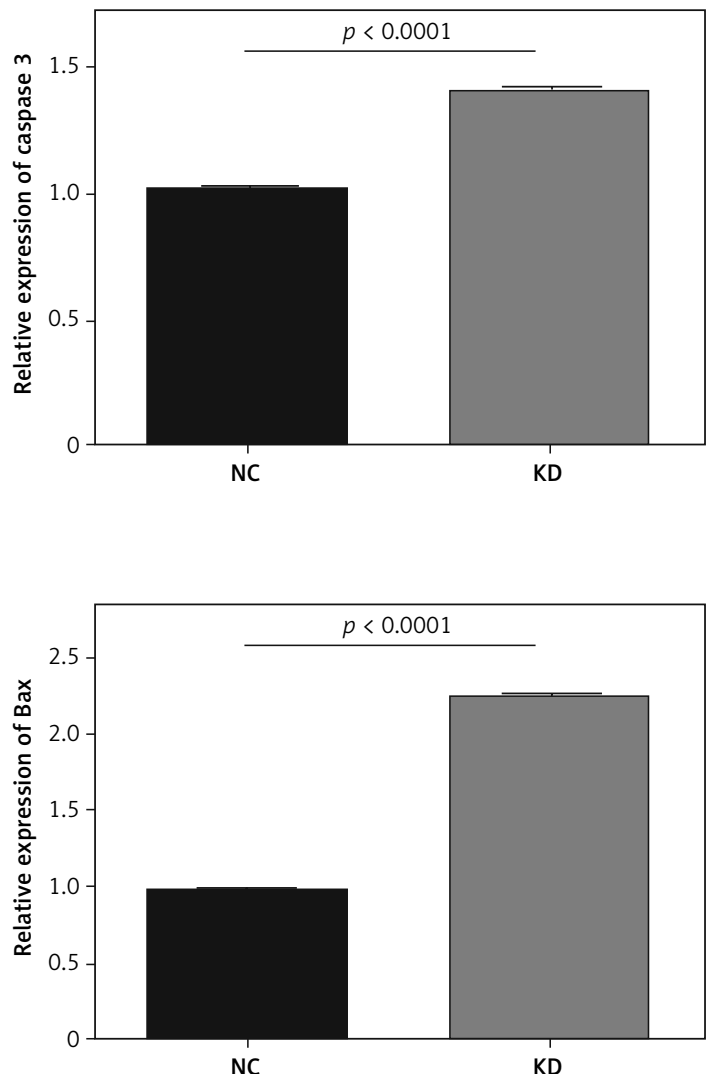

NC
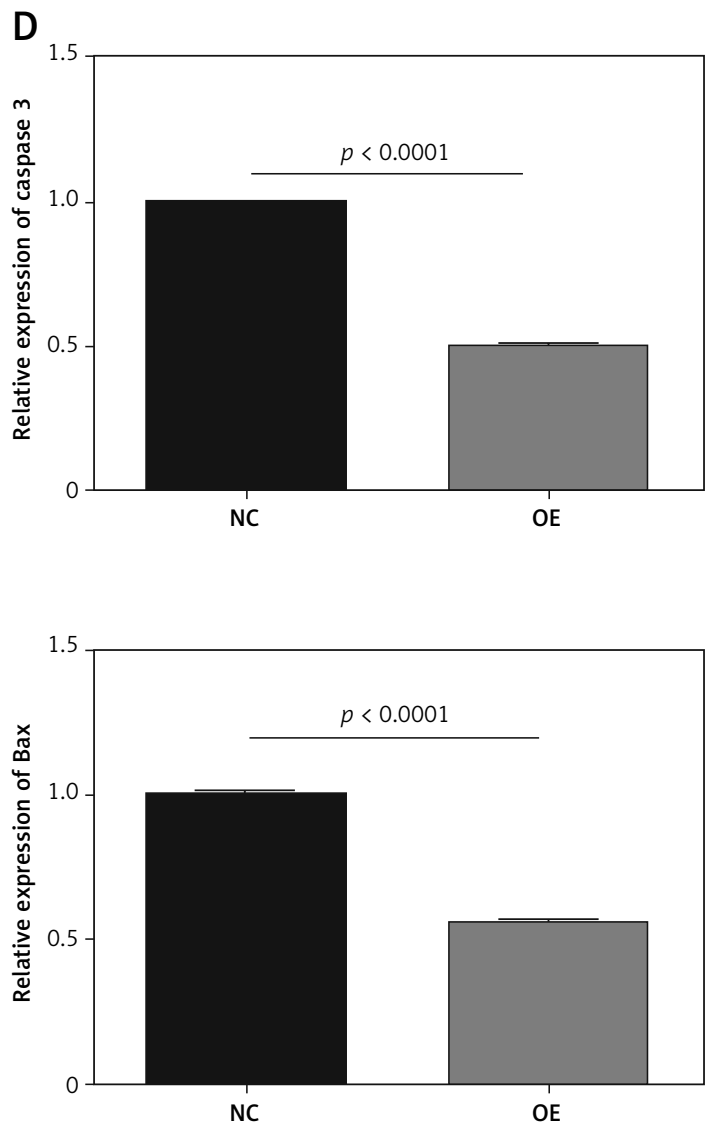

Figure 4. Upregulated FoxO3a expression suppressed apoptotic proteins in neuronal cells in response to hypoxia/ ischaemia. A, B - Relative expression levels of caspase-3, caspase-9, and Bax in B35 cells with or without FoxO3a knockdown treated with $\mathrm{CoCl}_{2}$ for 72 h. C, D - Relative expression levels of caspase-3, caspase-9, and Bax in B35 cells with or without FoxO3a overexpression treated with $\mathrm{CoCl}_{2}$ for $72 \mathrm{~h}$ 
A

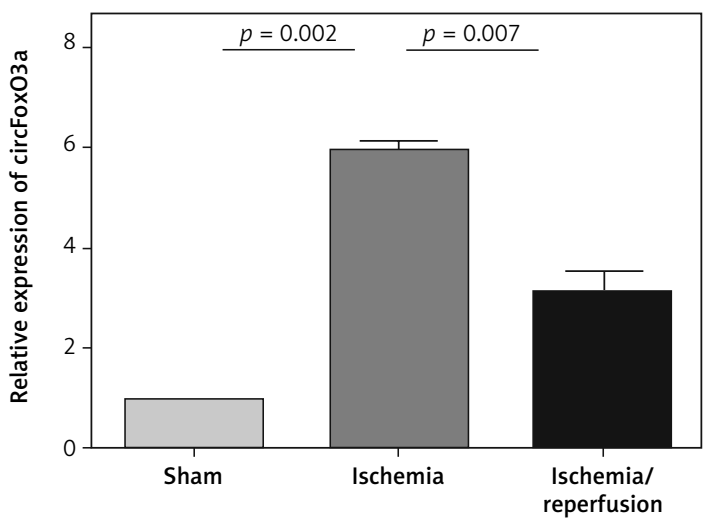

C

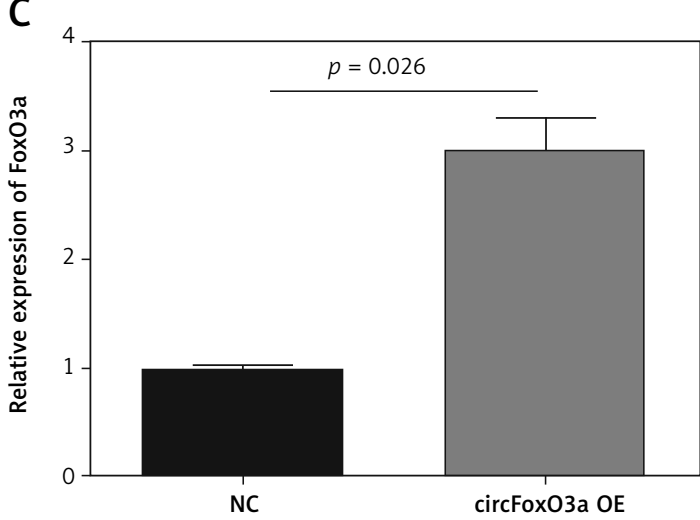

B

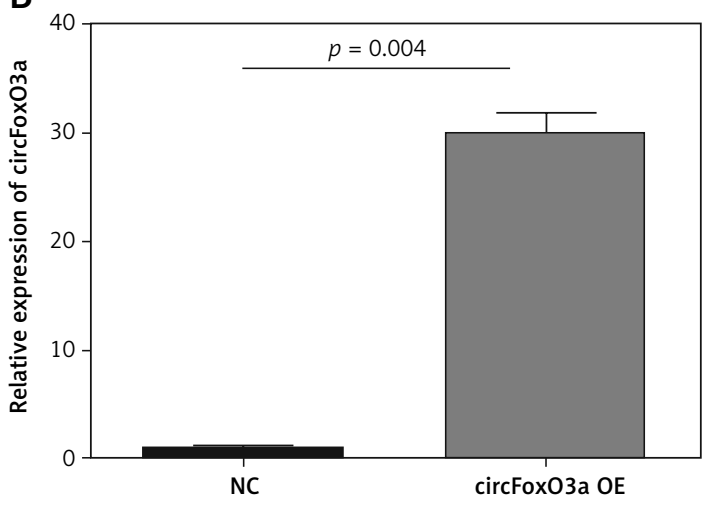

D
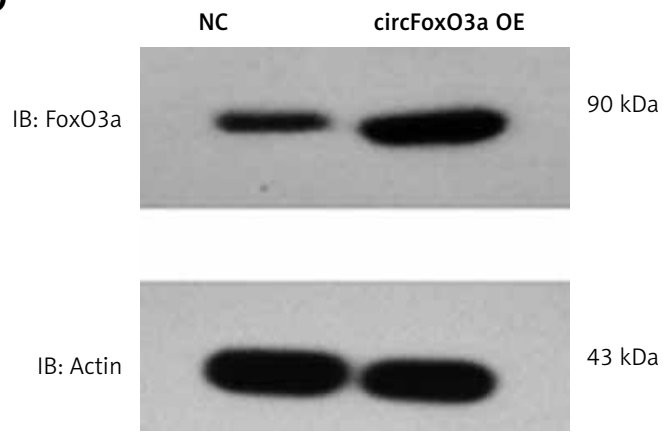

Figure 5. The upregulation of circFoxO3 enhanced FoxO3a expression levels in neuronal cells. A - Relative expression levels of circFoxO3 in the sham, MCAO, and MCAO/reperfusion groups. B - Relative expression levels of circFoxO3 in the NC and circFoxO3 OE cells. C, D - Relative expression levels of FoxO3a in $\mathrm{B} 35$ cells with or without circFoxO3 overexpression

mia/hypoxia exposure. Furthermore, the suppression of FoxO3a expression in $\mathrm{B} 35$ cells significantly increased hypoxic cell apoptosis. In contrast, the overexpression of FoxO3a significantly reduced hypoxic cell apoptosis. As expected, circFoxO3 and FoxO3a had consistent variation trends during ischaemia/hypoxia exposure, with the underlying mechanisms to be revealed in future work.

FoxO3a was reported to exhibit stronger immunostaining in the cytoplasm of breast cancer cells than in benign breast tissues, which predicted poor survival [15]. In acute myeloid leukaemia, the viability of leukaemia stem cells depended on the expression of FoxO3a [40]. These complex observations underscore the complicated dual functions of FoxO3a [40]. In the field of tumour targeted therapy, it was proven that targeting the non-canonical AKT-FoxO3a axis turned out to be a novel therapeutic strategy for treating oral squamous cell carcinoma (OSCC) patients [41]. In experimental colon cancer, quinazoline-based small molecule suppressed tumour progression by inhibiting PI3K/Akt/FoxO3a signalling pathway [42]. In this study, we revealed the expression and superficial functions of FoxO3a in cerebral ischae$\mathrm{mia} /$ reperfusion. The precise signalling pathway of FoxO3a in regulating ischaemia/hypoxia-induced apoptosis needs to be explored in order to identify clinical potential of FoxO3a (or other molecules in the pathway) as a neuroprotective agent.

Several studies have shown that FoxO3a protects cells from apoptosis and promotes invasion and metastasis under stress conditions through the regulation of the nuclear factor $\kappa \mathrm{B}(\mathrm{NF}-\kappa \mathrm{B})$ signalling pathway and the transcriptional regulation of genes involved in cell migration [8, 21, 43-45]. Consistently, our study revealed that upregulated FoxO3a expression protected neuronal cells from hypoxia/ischaemia-induced apoptosis via inactivation of the apoptotic pathway. However, some limitations have to be addressed. Although a relationship between FoxO3a and hypoxic apoptosis has previously been reported $[29,44]$, the specific mechanisms of FoxO3a in protecting neuronal cells under ischaemia/hypoxia stress still need further investigation.

The mechanism underlying the biological function of circFoxO3 is currently unknown. As shown in previous studies, circRNAs may act as endogenous sponges to interact with miRNAs and therefore regulate the expression of miRNA target genes. For instance, circRNA TLK1 func- 
tions as a miR-355 sponge to aggravate neuronal injury and neurological deficits after ischaemic stroke [46]. It is worth noting that circFoxO3 exerts pro-tumourigenic activity in glioblastoma by sponging miR-138-5p /miR-432-5p, which promotes expression of target gene named nuclear factor of activated T cells (NFAT5) [36]. In accordance with these findings, there is a possibility that circFoxO3 also functions as miRNA sponge to regulate neuronal injury during ischaemia/hypoxia. To prove this hypothesis, deep database mining and sequence alignment analysis may be optional strategies for discovering potential miRNA targets of circFoxO3. The interaction between circFoxO3 and FoxO3a, which have consistently changing trends during cerebral ischaemia/hypoxia, needs to be verified in future studies as well.

Because there are several signalling pathways involved in the rat model of ischemia/reperfusion injury [47], especially the neuroinflammation [48] and PI3K-Akt pathway [47, 49-51], the effect of circFoxO3 and FoxO3a might have an effect also on other pathways. Therefore, future studies are needed to elucidate the specific role of FoxO3a.

In conclusion, our study is the first to establish that upregulated FoxO3a expression protects neuronal cells from hypoxia/ischaemia-induced apoptosis via activation of the apoptotic pathway. The therapeutic potential of FoxO3a and (or) circFoxO3 as neuroprotective agents is worth exploring.

\section{Acknowledgments}

These authors (Rongjia Lu, Huajie Cai, Yu Liao, Keman Liao) contributed equally to this work.

\section{Conflict of interest}

The authors declare no conflict of interest.

\section{References}

1. Heron M. Deaths: Leading Causes for 2015. National vital statistics reports: from the Centers for Disease Control and Prevention, National Center for Health Statistics, National Vital Statistics System. 2017; 66: 1-76.

2. Doyle KP, Simon RP, Stenzel-Poore MP. Mechanisms of ischemic brain damage. Neuropharmacology 2008; 55: 310-8.

3. Dirnagl U, Iadecola C, Moskowitz MA. Pathobiology of ischaemic stroke: an integrated view. Trends Neurosci 1999; 22: 391-7.

4. Khoshnam SE, Winlow W, Farzaneh M, Farbood Y, Moghaddam HF. Pathogenic mechanisms following ischemic stroke. Neurol Sci 2017; 38: 1167-86.

5. Sekerdag E, Solaroglu I, Gursoy-Ozdemir Y. Cell death mechanisms in stroke and novel molecular and cellular treatment options. Curr Neuropharmacol 2018; 16: 1396-415.

6. Cheon SY, Kim EJ, Kim JM, Koo BN. Cell type-specific mechanisms in the pathogenesis of ischemic stroke: the role of apoptosis signal-regulating kinase 1. Oxid Med Cell Longev 2018; 2018: 2596043.

7. Puyal J, Ginet V, Clarke PG. Multiple interacting cell death mechanisms in the mediation of excitotoxicity and ischemic brain damage: a challenge for neuroprotection. Progress Neurobiol 2013; 105: 24-48.

8. Kops GJ, Dansen TB, Polderman PE, et al. Forkhead transcription factor $\mathrm{FOXO} 3$ a protects quiescent cells from oxidative stress. Nature 2002; 419: 316-21.

9. Gomes AR, Zhao F, Lam EW. Role and regulation of the forkhead transcription factors FOXO3a and FOXM1 in carcinogenesis and drug resistance. Chin J Cancer 2013; 32: $365-70$

10. Skurk C, Maatz H, Kim HS, et al. The Akt-regulated forkhead transcription factor FOXO3a controls endothelial cell viability through modulation of the caspase- 8 inhibitor FLIP. J Biol Chem 2004; 279: 1513-25.

11. Das TP, Suman S, Alatassi H, Ankem MK, Damodaran C. Inhibition of AKT promotes FOXO3a-dependent apoptosis in prostate cancer. Cell Death Dis 2016; 7: e2111.

12. Shaw RJ, Cantley LC. Ras, PI(3)K and mTOR signalling controls tumour cell growth. Nature 2006; 441: 424-30.

13. Sanphui P, Biswas SC. FoxO3a is activated and executes neuron death via Bim in response to beta-amyloid. Cell Death Dis 2013; 4: e625.

14. Murtaza G, Khan AK, Rashid R, Muneer S, Hasan SMF, Chen J. FOXO transcriptional factors and long-term living. Oxid Med Cell Longev 2017; 2017: 3494289.

15. Hu MC, Lee DF, Xia W, et al. IkappaB kinase promotes tumorigenesis through inhibition of forkhead FOXO3a. Cell 2004; 117: 225-37.

16. Tenbaum SP, Ordonez-Moran P, Puig I, et al. Beta-catenin confers resistance to PI3K and AKT inhibitors and subverts $\mathrm{FOXO3a}$ to promote metastasis in colon cancer. Nat Med 2012; 18: 892-901.

17. Brunet A, Bonni A, Zigmond MJ, et al. Akt promotes cell survival by phosphorylating and inhibiting a Forkhead transcription factor. Cell 1999; 96: 857-68.

18. Yang JY, Zong CS, Xia W, et al. ERK promotes tumorigenesis by inhibiting FOXO3a via MDM2-mediated degradation. Nat Cell Biol 2008; 10: 138-48.

19. Prabhu VV, Allen JE, Dicker DT, El-Deiry WS. Small-molecule ONC201/TIC10 targets chemotherapy-resistant colorectal cancer stem-like cells in an Akt/Foxo3a/TRAIL-dependent manner. Cancer Res 2015; 75: 1423-32.

20. Sunayama J, Sato A, Matsuda K, et al. FoxO3a functions as a key integrator of cellular signals that control glioblastoma stem-like cell differentiation and tumorigenicity. Stem Cells 2011; 29: 1327-37.

21. Ni D, Ma X, Li HZ, et al. Downregulation of FOXO3a promotes tumor metastasis and is associated with metastasis-free survival of patients with clear cell renal cell carcinoma. Clin Cancer Res 2014; 20: 1779-90.

22. Reagan-Shaw S, Ahmad N. RNA interference-mediated depletion of phosphoinositide 3-kinase activates forkhead box class $\mathrm{O}$ transcription factors and induces cell cycle arrest and apoptosis in breast carcinoma cells. Cancer Res 2006; 66: 1062-9.

23. Chen YF, Pandey S, Day $\mathrm{CH}$, et al. Synergistic effect of HIF-1alpha and FoxO3a trigger cardiomyocyte apoptosis under hyperglycemic ischemia condition. J Cell Physiol 2018; 233: 3660-71.

24. Feng CC, Lin CC, Lai YP, et al. Hypoxia suppresses myocardial survival pathway through HIF-1alpha-IGFBP-3-dependent signaling and enhances cardiomyocyte autophagic and apoptotic effects mainly via FoxO3a-induced BNIP3 expression. Growth Factors 2016; 34: 73-86. 
25. Sengupta A, Molkentin JD, Paik JH, DePinho RA, Yutzey KE. FoxO transcription factors promote cardiomyocyte survival upon induction of oxidative stress. J Biol Chem 2011; 286: 7468-78.

26. Tia N, Singh AK, Pandey P, Azad CS, Chaudhary P, Gambhir IS. Role of Forkhead Box O (FOXO) transcription factor in aging and diseases. Gene 2018; 648: 97-105.

27. Xiao Q, Ye Q, Wang W, et al. Mild hypothermia pretreatment protects against liver ischemia reperfusion injury via the PI3K/AKT/FOXO3a pathway. Mol Med Rep 2017; 16: 7520-6.

28. Alexiou K, Wilbring M, Matschke K, Dschietzig T. Relaxin protects rat lungs from ischemia-reperfusion injury via inducible NO synthase: role of ERK-1/2, PI3K, and forkhead transcription factor FKHRL1. PLoS One 2013; 8: e75592.

29. Li D, Li X, Wu J, et al. Involvement of the JNK/FOXO3a/ Bim pathway in neuronal apoptosis after hypoxic-ischemic brain damage in neonatal rats. PloS One 2015; 10: e0132998.

30. Li D, Luo L, Xu M, et al. AMPK activates FOXO3a and promotes neuronal apoptosis in the developing rat brain during the early phase after hypoxia-ischemia. Brain Res Bull 2017; 132: 1-9.

31. Li D, Qu Y, Mao M, et al. Involvement of the PTEN-AKTFOXO3a pathway in neuronal apoptosis in developing rat brain after hypoxia-ischemia. J Cereb Blood Flow Metab 2009; 29: 1903-13.

32. Yoo KY, Kwon SH, Lee CH, et al. FoxO3a changes in pyramidal neurons and expresses in non-pyramidal neurons and astrocytes in the gerbil hippocampal CA1 region after transient cerebral ischemia. Neurochem Res 2012; 37: 588-95.

33. Zhan L, Wang T, Li W, Xu ZC, Sun W, Xu E. Activation of Akt/FoxO signaling pathway contributes to induction of neuroprotection against transient global cerebral ischemia by hypoxic pre-conditioning in adult rats. J Neurochem 2010; 114: 897-908.

34. Lu WY. Roles of the circular RNA circ-Foxo3 in breast cancer progression. Cell Cycle 2017; 16: 589-90.

35. Zhang Y, Zhao H, Zhang L. Identification of the tumorsuppressive function of circular RNA FOXO3 in nonsmall cell lung cancer through sponging miR155. Mol Med Rep 2018; 17: 7692-700.

36. Zhang S, Liao K, Miao Z, et al. CircFOXO3 promotes glioblastoma progression by acting as a competing endogenous RNA for NFAT5. Neurooncology 2019; 21: 1284-96.

37. Yang W, Du WW, Li X, Yee AJ, Yang BB. Foxo3 activity promoted by non-coding effects of circular RNA and Foxo3 pseudogene in the inhibition of tumor growth and angiogenesis. Oncogene 2016; 35: 3919-31.

38. Du WW, Yang W, Chen Y, et al. Foxo3 circular RNA promotes cardiac senescence by modulating multiple factors associated with stress and senescence responses. Eur Heart J 2017; 38: 1402-12.

39. Yang YF, Chen Z, Hu SL, et al. Interleukin-1 receptor associated kinases-1/4 inhibition protects against acute hypoxia/ischemia-induced neuronal injury in vivo and in vitro. Neuroscience 2011; 196: 25-34.

40. Sykes SM, Lane SW, Bullinger L, et al. AKT/FOXO signaling enforces reversible differentiation blockade in myeloid leukemias. Cell 2011; 146: 697-708.

41. Liu Y. Targeting the non-canonical AKT-FOXO3a axis: A potential therapeutic strategy for oral squamous cell carcinoma. EBioMedicine 2019; 49: 6-8.
42. Oazi AK, Hussain A, Khan S, et al. Ouinazoline based small molecule exerts potent tumour suppressive properties by inhibiting $\mathrm{PI3K} / \mathrm{Akt} /$ FoxO3a signalling in experimental colon cancer. Cancer Letters 2015; 359: 47-56.

43. Li Z, Zhang H, Chen Y, Fan L, Fang J. Forkhead transcription factor $\mathrm{FOXO} 3 \mathrm{a}$ protein activates nuclear factor kappaB through B-cell lymphoma/leukemia 10 (BCL10) protein and promotes tumor cell survival in serum deprivation. J Biol Chem 2012; 287: 17737-45.

44. Bakker WJ, Harris IS, Mak TW. FOXO3a is activated in response to hypoxic stress and inhibits HIF1-induced apoptosis via regulation of CITED2. Mol Cell 2007; 28 941-53.

45. Storz P, Doppler H, Copland JA, Simpson KJ, Toker A. FOXO3a promotes tumor cell invasion through the induction of matrix metalloproteinases. Mol Cell Biol 2009; 29: 4906-17.

46. Wu F, Han B, Wu S, et al. Circular RNA aggravates neuronal injury and neurological deficits after ischemic stroke via miR-335-3p/TIPARP. J Neurosci 2019; 39: 7369-93.

47. Eken MK, Ersoy GS, Kaygusuz El, et al. Etanercept protects ovarian reserve against ischemia/reperfusion injury in a rat model. Arch Med Sci 2019; 15: 1104-12.

48. Yang L, Jiang L, Jiang D, Liu B, Jin S. The protective effects of salvianolic acid A against hepatic ischemia-reperfusion injury via inhibiting expression of toll-like receptor 4 in rats. Arch Med Sci 2019; 15: 1599-607.

49. Ying C, Wang S, Lu Y, et al. Glucose fluctuation increased mesangial cell apoptosis related to AKT signal pathway. Arch Med Sci 2019; 15: 730-7.

50. Guo H, He Y, Bu C, Peng Z. Antitumor and apoptotic effects of 5-methoxypsoralen in U87MG human glioma cells and its effect on cell cycle, autophagy and PI3K/ Akt signaling pathway. Arch Med Sci 2019; 15: 1530-8.

51. Zhang Y, Zhang R, Ni H. Eriodictyol exerts potent anticancer activity against $\mathrm{A} 549$ human lung cancer cell line by inducing mitochondrial-mediated apoptosis, G2/M cell cycle arrest and inhibition of m-TOR/PI3K/Akt signalling pathway. Arch Med Sci 2020; 16: 446-52. 